\title{
Anemia ferropriva e alimentação no segundo ano de vida no Rio de Janeiro, Brasil ${ }^{1}$
}

\author{
Elisa Lacerda² e Antonio José Cunha ${ }^{3}$
}

RESUMO Objetivos. Avaliar a influência de práticas alimentares na incidência de anemia em lactentes a partir do estudo do perfil alimentar das crianças atendidas em um ambulatório de pediatria no Rio de Janeiro, Brasil.

Métodos. Trata-se de um estudo transversal com 288 lactentes de 12 a 18 meses que compareceram ao ambulatório de janeiro a junho de 1993. As crianças foram avaliadas quanto à presença de anemia ferropriva; além disso, foi realizado um recordatório de 24 horas e um registro de freqüência de consumo de alimentos ricos em ferro junto aos responsáveis pelas crianças.

Resultados. Cento e quarenta e quatro crianças apresentavam anemia (hemoglobina $<11$ $\mathrm{g} / \mathrm{dL}$ ); destas, 38 apresentavam anemia grave (hemoglobina $<9,5 \mathrm{~g} / \mathrm{dL}$ ). Encontrou-se baixo consumo de ferro biodisponível, consumo de vitamina $C$ dissociado das refeições e baixo consumo de carne. Observou-se associação significativa entre a prevalência de anemia severa e o consumo inadequado de ferro (razão de prevalência $=2,28$; intervalo de confiança $=1,12-4,66$; $\mathrm{P}=0,02)$. O consumo de ferro biodisponível foi maior no grupo sem anemia $(\mathrm{P}=0,04)$.

Conclusões. Os responsáveis pelos lactentes devem ser informados sobre a composição nutricional da alimentação complementar a fim de aumentar a biodisponibilidade de ferro na dieta da criança. A estratégia da Atenção Integrada às Doenças Prevalentes na Infância pode contribuir neste sentido.

Palavras-chave Anemia ferropriva, alimentação complementar, lactente.

A deficiência de ferro é, isoladamente, a mais comum das deficiências nutricionais no mundo, sendo a anemia a sua forma mais severa. Os lac-

\footnotetext{
Estudo financiado pelo Instituto de Puericultura e Pediatria Martagão Gesteira, Rio de Janeiro, Brasil, e The Health Foundation, Nova Iorque, Estados Unidos da América.

2 Instituto de Nutrição, Universidade Federal do Rio de Janeiro (UFRJ), e Centro de Referência César Pernetta, Rio de Janeiro (RJ), Brasil. Correspondência e pedidos de separatas devem ser enviados a esta autora no seguinte endereço: Rua Cosme Velho 415/305, CEP 22241-090, Rio de Janeiro, RJ, Brasil. Telefone: +55-21-2053784; fax: +55-21-2808343; e-mail: elisalacerda@ufrj.br

3 Departamento de Pediatria da Faculdade de Medicina e Instituto de Puericultura e Pediatria Martagão Gesteira, UFRJ, e Centro de Referência César Pernetta, Rio de Janeiro (RJ) Brasil.
}

tentes fazem parte do grupo de risco para o desenvolvimento da anemia ferropriva, com uma prevalência global estimada em 43\%, devido à maior necessidade de ferro em função do crescimento acentuado dos tecidos $(1,2)$. No Brasil, estudos setorizados revelam aumento da prevalência de anemia ao longo dos anos (3-6). A anemia ferropriva tem como causa imediata o consumo insuficiente de alimentos fontes de ferro ou a baixa biodisponibilidade do ferro ingerido. Na infância, constitui um grave problema de saúde pública devido à elevada prevalência e distribuição e às significativas repercussões no desenvolvimento psicomotor da criança afetada $(7,8)$.
Vários estudos destacam a importância da composição da dieta da criança para a manutenção de um adequado estado nutricional de ferro. Dentre os fatores que potencializam a absorção de ferro encontram-se o ácido ascórbico, ácidos orgânicos, carnes, vitamina A e beta-caroteno (9-13). Dentre os fatores inibidores da $a b-$ sorção de ferro encontram-se polifenóis, fitatos e outros elementos inorgânicos (11, 14-19). Estudos em seres humanos mostraram que o cálcio chega a reduzir a absorção de ferro em até $60 \%$, sendo recomendada a redução no consumo de alimentos lácteos junto às refeições contendo ferro heme (de origem animal), especial- 
mente no caso de indivíduos que consomem muitos alimentos lácteos e têm alta necessidade de ferro, como os lactentes (20-23).

Um estudo realizado na Argentina (24) revelou uma incidência de 47\% de anemia e uma associação com o baixo consumo de ferro (5,6 mg ao dia), a introdução precoce de leite de vaca e o baixo consumo de alimentos enriquecidos com ferro. No Brasil, um estudo no Município de São Paulo revelou que $49 \%$ das dietas de crianças eram deficientes em ferro, com valores muito abaixo da ingestão dietética recomendada (IDR) do nutriente (25). Outro estudo indicou a necessidade de suplementação profilática de ferro durante o $1^{\circ}$ ano de vida, em função de $44 \%$ das dietas de crianças menores de 2 anos não terem atingido a IDR para ferro (3). Uchimura (26), estudando escolares de Maringá (Estado do Paraná), encontrou $32 \%$ de anemia e associação com o baixo consumo de feijão e carnes e a ausência de frutas nas refeições. Novamente no Município de São Paulo, Sichieri et al. (6) observaram consumo de 6,2 mg de ferro por dia, enquanto que, em região rural do Estado da Bahia, Silva (27) observou consumo de 7,3 mg ferro por dia; ambos os estudos foram realizados com crianças na faixa etária de 12 a 24 meses.

Visando contribuir para a prevenção da anemia ferropriva em crianças menores de 1 ano atendidas em unidades de saúde, e em decorrência da escassez de estudos dietéticos em nosso meio, procurou-se determinar os fatores alimentares associados à anemia ferropriva em crianças de um hospital pediátrico universitário. Estudou-se o teor de energia, proteína, cálcio, ferro, ferro biodisponível e vitamina $\mathrm{C}$ das refeições; os tipos e combinações de alimentos; a freqüência de consumo de alimentos ricos em ferro; e a associação desses fatores com a presença de anemia ferropriva.

\section{MATERIAIS E MÉTODOS}

Trata-se de um estudo descritivo transversal, realizado no Instituto de
Puericultura e Pediatria Martagão Gesteira (IPPMG) da Universidade Federal do Rio de Janeiro (UFRJ). O IPPMG é um hospital de referência, atendendo prioritariamente a clientela de uma área contendo 28 bairros e 58 favelas do município do Rio de Janeiro (área programática 3.1, conforme divisão administrativa do setor saúde municipal). Possui dois grandes setores de atendimento pediátrico externo geral (não especializado), onde o presente estudo foi realizado. Foram incluídas todas as crianças de 12 a 18 meses que compareceram a estes setores no período de janeiro a junho de 1993, totalizando 350 crianças. Foram excluídas as crianças portadoras de anemia de outra etiologia sabidamente não ferropriva (hemoglobinopatias, anemias hemolíticas e anemia megaloblástica), as que haviam recebido hemotransfusão nos 3 meses anteriores à pesquisa, as portadoras de doenças crônicas (diarréias, doenças renais, imunodeficiências, paralisias cerebrais e cardiopatias congênitas cianóticas) e as que tiveram menos de três consultas no IPPMG, reduzindo a amostra em 24 crianças. Também foram excluídas 38 crianças cujos responsáveis se recusaram a participar da pesquisa.

As informações sobre a dieta da criança foram obtidas por um dos autores (EMAL) e entrevistadores treinados, mediante entrevista com a pessoa que acompanhava a criança na visita ao hospital. Foram realizados um recordatório de 24 horas e um registro de freqüência de consumo de alimentos ricos em ferro. O recordatório de 24 horas registrou o consumo alimentar da criança no dia anterior ao da entrevista, com objetivo de avaliar o consumo de energia, proteínas, ferro, ferro biodisponível, cálcio e vitamina C. Foi utilizado um instrumento ilustrado com desenhos de copos e talheres a fim de facilitar a resposta dos entrevistados. A quantidade dos alimentos foi anotada na forma de medida caseira (por exemplo, unidade, colher, copo).

O consumo de nutrientes e ferro biodisponível (Metodologia Mosen) foi calculado utilizando-se o programa SISNUT (28). O consumo adequado foi definido como valores iguais ou superiores à IDR e o consumo inadequado como valores inferiores à IDR (29-31). Foram analisados os recordatórios de 24 horas de crianças cujos acompanhantes permaneceram pelo menos 10 turnos de 8 horas semanais junto à criança, assumindo-se que tempo inferior a este não permitiria respostas adequadas. Entretanto, em 10 casos, o entrevistado não se julgou apto a responder o questionário ou o entrevistador julgou que o entrevistado não estava apto a respondê-lo, de forma que o recordatório de 24 horas não foi analisado. Também foram descartados 40 recordatórios de 24 horas cujo relato não refletia a alimentação habitual da criança e 28 com preenchimento inadequado, totalizando 78 perdas e uma análise de 210 recordatórios.

Foi obtida a freqüência de consumo de alimentos ricos em ferro no momento do estudo (fígado, carnes, feijão, ovo, gema de ovo e hortaliças folhosas) e os resultados foram categorizados da seguinte forma: alimentos consumidos uma a duas vezes ao dia (diariamente), alimentos consumidos quatro a seis vezes por semana (freqüentemente), alimentos consumidos uma a três vezes por semana (pouco), alimentos consumidos menos do que uma vez por semana (raramente) e alimentos nunca oferecidos. Para a avaliação da associação entre a freqüência de consumo e a anemia, criaram-se duas categorias: freqüência adequada (duas vezes ao dia até quatro vezes por semana) e freqüência inadequada (inferior a quatro vezes por semana). Esta determinação foi baseada nas recomendações usuais sobre alimentação infantil a fim de atender à IDR dos nutrientes. No caso do fígado, também foi considerada freqüência adequada o consumo de uma a três vezes por semana. Não foram analisadas as freqüências de consumo das 10 crianças cujos acompanhantes não estavam aptos a responder, totalizando uma análise de 278 freqüências de consumo de alimentos ricos em ferro.

No mesmo dia, após a entrevista, foi colhido sangue e a hemoglobina foi 
dosada no IPPMG pelo método da cianometahemoglobina. Foram consideradas com anemia ferropriva as crianças com níveis de hemoglobina $<11 \mathrm{~g} / \mathrm{dL}$; e com anemia grave, as crianças com hemoglobina $<9,5 \mathrm{~g} / \mathrm{dL}$ (2). Os dados foram armazenados e analisados utilizando-se o programa Epi Info 6.04 (32). As variáveis contínuas foram apresentadas sob forma de medidas de tendência central (média aritmética e média aritmética de dados agrupados em intervalos) e medida de dispersão (desvio padrão, DP). Utilizou-se o teste não-paramétrico de Mann-Whitney para comparação de médias quando as variâncias não eram homogêneas. Para a inferência estatística das medianas utilizou-se o teste de Wilcoxon. Para a comparação estatística das proporções utilizou-se o teste do qui-quadrado $\left(\chi^{2}\right)$. Estabeleceu-se um nível de significância de 5\%. Foi avaliada a associação entre as variáveis categóricas e a presença de anemia utilizando a razão de prevalências (RP). Para indicar a precisão da estima-

TABELA 1. Características de 288 crianças de 12 a 18 meses, estudo sobre anemia ferropriva e alimentação, Rio de Janeiro, Brasil, 1993

\begin{tabular}{lrr}
\hline \multicolumn{1}{c}{ Características } & No. & $\%$ \\
\hline Idade & & \\
12 meses & 70 & 24 \\
13 meses & 41 & 14 \\
14 meses & 42 & 15 \\
15 meses & 41 & 14 \\
16 meses & 33 & 12 \\
17 meses & 31 & 11 \\
18 meses & 30 & 10 \\
Sexo & & \\
$\quad$ Masculino & 155 & 54 \\
Feminino & 133 & 46 \\
Hemoglobina & 38 & 13 \\
$\quad<9,5$ g/dL & 144 & 50 \\
$\geq 11$ g/dL & 106 & 37 \\
$\quad \geq 9,5$ e < 11 g/dL & & \\
Acompanhantes & 275 & 96 \\
$\quad$ Mãe & 13 & 5 \\
Outros (pai, tios, avós) & & \\
Estado nutricional (peso/ & & \\
$\quad$ comprimento) & 260 & 91 \\
$\quad \geq-2$ desvios padrão & 25 & 9 \\
$\quad<-2$ desvios padrão & & \\
\hline
\end{tabular}

tiva da RP, foi calculado o intervalo de confiança (IC) de $95 \%$. O estudo foi aprovado pela Comissão de Ética Médica do IPPMG e houve consentimento por escrito dos participantes.

\section{RESULTADOS}

Dentre as 350 crianças elegíveis, ocorreram 62 perdas ocasionadas pela discordância dos responsáveis em participar do estudo $(n=38)$ e presença de critérios de exclusão $(n=24)$. A amostra final foi composta de 288 crianças, das quais $95 \%$ eram acompanhadas pelas mães e $5 \%$ por pais, tios ou avós. A média de idade da amostra foi de 14,5 meses $(\mathrm{DP}=2)$. A prevalência de anemia foi de $50 \%(\mathrm{n}=144)$, sendo $13 \%(\mathrm{n}=38)$ a de anemia grave. A maior parte da amostra encontrava-se em bom estado nutricional (tabela 1).

A tabela 2 apresenta o consumo médio de nutrientes dos 210 recordatórios de 24 horas analisados e a adequação à IDR. As características gerais das 78 crianças cujos recordatórios de 24 horas foram descartados não diferiram da amostra analisada. Observouse consumo protéico adequado para todas as crianças; o consumo de energia, cálcio e vitamina $C$ era adequado na maioria das crianças. Foi encontrado baixo consumo de ferro e ferro biodisponível. O percentual médio de ferro biodisponível em relação ao consumo total de ferro foi de $7 \%$, caracte- rizando uma dieta de baixa biodisponibilidade de ferro (30).

Considerando a freqüência de anemia, o consumo de ferro e de ferro biodisponível em crianças sem anemia foi 10 e $19 \%$ superior ao consumo em crianças com anemia $(P=0,04)$, respectivamente (tabela 3 ). As crianças com consumo inadequado de ferro tinham 2,28 vezes mais chances de ter anemia grave quando comparadas com as crianças com consumo adequado de ferro ( $\mathrm{RP}=2,28$; IC $=1,12$ $4,66)$, sendo essa associação estatisticamente significativa $(P=0,02)$. A chance de ter anemia grave foi 1,71 vez maior em crianças com consumo inadequado de ferro biodisponível quando comparadas com crianças com consumo adequado de ferro biodisponível ( $\mathrm{RP}=1,71$; IC = 0,71-4,15). Entretanto, esta associação não foi estatisticamente significativa (tabela 4).

A análise do consumo de nutrientes por refeição demonstrou que as pequenas refeições eram ricas em cálcio e energia e pobres em ferro e vitamina C. As grandes refeições (almoço e jantar) eram ricas em energia, proteína, ferro, ferro biodisponível, vitamina $\mathrm{Ce}$ pobres em cálcio (tabela 5). Os alimentos mais consumidos no desjejum foram leite de vaca (fluido ou em pó) e cereal (enriquecido ou não). Os menos consumidos foram frutas, pães e biscoitos. Na colação, a fruta foi o alimento mais consumido. O lanche revelou freqüência elevada de leite de

TABELA 2. Consumo médio diário de nutrientes e adequação aos valores dietéticos de referência em 210 crianças de 12 a 18 meses, Rio de Janeiro, Brasil, 1993

\begin{tabular}{|c|c|c|c|c|c|}
\hline \multirow[b]{2}{*}{ Nutriente } & \multirow{2}{*}{$\begin{array}{l}\text { Média (desvio } \\
\text { padrão) }\end{array}$} & \multicolumn{2}{|c|}{$\begin{array}{c}\text { Consumo } \\
\text { inadequado }^{\mathrm{a}}\end{array}$} & \multicolumn{2}{|c|}{$\begin{array}{l}\text { Consumo } \\
\text { adequado }\end{array}$} \\
\hline & & No. & $\%$ & No. & $\%$ \\
\hline Energia (kcal) & $146,6 \quad(433)$ & 45 & 22 & 162 & 78 \\
\hline Proteína $(\mathrm{g})$ & $56,6 \quad(19,3)$ & 0 & 0 & 207 & 100 \\
\hline Ferro (mg) & $10,7 \quad(4,6)$ & 100 & 48 & 110 & 52 \\
\hline Ferro biodisponível (mg) & $(0,5)$ & 158 & 75 & 52 & 25 \\
\hline Cálcio (mg) & 968 (436) & 28 & 13 & 182 & 87 \\
\hline Vitamina C (mg) & $96 \quad(79)$ & 14 & 7 & 196 & 93 \\
\hline
\end{tabular}


TABELA 3. Consumo médio diário de nutrientes segundo freqüência de anemia em 210 crianças de 12 a 18 meses, Rio de Janeiro, Brasil, 1993

\begin{tabular}{lccc}
\hline & \multicolumn{2}{c}{ Anemia } & \\
\cline { 2 - 3 } \multicolumn{1}{c}{ Nutriente } & $\begin{array}{c}\text { Presente } \\
(\mathrm{n}=109)\end{array}$ & $\begin{array}{c}\text { Ausente } \\
(\mathrm{n}=101)\end{array}$ & $P$ \\
\hline Energia (kcal) & 1458 & 1478 & 0,44 \\
Proteína (g) & 54,1 & 58,9 & 0,06 \\
Ferro (mg) & 10,2 & 11,2 & 0,14 \\
Ferro biodisponível (mg) & 0,684 & 0,811 & 0,04 \\
Ferro animal (mg) & 2,044 & 2,535 & 0,06 \\
Cálcio (mg) & 957 & 984 & 0,72 \\
Vitamina C (mg) & 84,9 & 107,2 & 0,08 \\
\hline
\end{tabular}

vaca, frutas e cereais. A ceia 1 (refeição realizada antes da criança dormir) apresentou alta freqüência de leite de vaca e cereal e a ceia 2 (refeição realizada de madrugada) apresentou alta freqüência de leite materno. Os alimentos mais consumidos no almoço e jantar foram o feijão preto, cereais (arroz e macarrão), frutas, carne bovina e tubérculos ou raízes. Não foi constatado consumo de chá, mate, café ou leite infantil modificado. Encontrou-se baixo consumo de hortaliças folhosas, fígado, outras leguminosas (excluindo o feijão), leite materno, leite de vaca, refrigerantes e doces. No jantar, observou-se aumento no consumo de leite de vaca e redução no consumo de feijão, ovo, peixe e fruta. A carne mais consumida foi a bovina e a menos consumida, a de peixe.

A tabela 6 apresenta a freqüência de anemia em função da combinação de alimentos das refeições, especialmente referente à presença de alimentos que notadamente inibem ou aumentam a absorção de ferro na refeição. As combinações encontradas para almoço e jantar foram muito semelhantes, sendo somente apresentada a análise referente ao almoço. A prevalência de anemia foi semelhante em crianças que consumiam refeições com carne e refeições sem carne. A razão de prevalências mostra que havia 1,48 e 1,49 vez mais chance de terem anemia as crianças que realizaram refeições contendo ferro não-heme sem associação de fruta e ferro não-heme com associação de leite de vaca, respectivamente. Estas associações, entretanto, não foram estatisticamente significativas.

A análise da freqüência de consumo de alimentos ricos em ferro (tabela 7) mostrou que $63 \%$ das 278 crianças para quem essa análise foi realizada consumiam feijão diariamente e que $13 \%$ nunca tinham recebido este alimento. $\mathrm{O}$ ovo inteiro aparece principalmente numa freqüência de uma a três vezes por semana. As carnes bovina e de frango eram consumidas, por cerca de $80 \%$ da amostra, de uma a três vezes por semana. Cerca de metade da amostra consumia fígado de uma a três vezes por semana e um quarto da amostra não recebia este alimento. As hortaliças folhosas apresentaram uma freqüência de consumo baixa. Cerca de metade da amostra não as consumia (tabela 7). A prevalência de anemia foi maior em crianças com consumo inadequado de carne e hortaliças folhosas. As crianças com consumo adequado de fígado tiveram maior prevalência de anemia. Entretanto, estas diferenças não foram estatisticamente significativas (tabela 8).

\section{DISCUSSÃO}

Inquéritos dietéticos como o do pre-

TABELA 4. Associação entre consumo de energia, ferro e ferro biodisponível e anemia grave em crianças de 12 a 18 meses de idade, Rio de Janeiro, Brasil, 1993

\begin{tabular}{|c|c|c|c|c|c|c|}
\hline \multirow{3}{*}{$\begin{array}{c}\text { Adequação da } \\
\text { freqüência de consumo }\end{array}$} & \multicolumn{4}{|c|}{ Anemia } & \multirow{3}{*}{$\begin{array}{c}\mathrm{RP}^{\mathrm{c}} \\
\text { (IC 95\%) }\end{array}$} & \multirow[b]{3}{*}{$P$} \\
\hline & \multicolumn{2}{|c|}{ Grave $^{a}$} & \multicolumn{2}{|c|}{ Ausente } & & \\
\hline & No. & $\%$ & No. & $\%$ & & \\
\hline \multicolumn{7}{|l|}{ Energia } \\
\hline Inadequado & 7 & 28 & 18 & 72 & 1,43 & 0,36 \\
\hline Adequado & 20 & 72 & 82 & 80 & $(0,68-3,00)$ & \\
\hline \multicolumn{7}{|l|}{ Ferro } \\
\hline Inadequado & 19 & 31 & 43 & 69 & 2,28 & 0,02 \\
\hline Adequado & 9 & 13 & 58 & 87 & $(1,12-4,66)$ & \\
\hline \multicolumn{7}{|l|}{ Ferro biodisponível } \\
\hline Inadequado & 23 & 24 & 71 & 75 & 1,71 & 0,21 \\
\hline Adequado & 5 & 14 & 30 & 86 & $(0,71-4,15)$ & \\
\hline
\end{tabular}
sente estudo apresentam limitações que devem ser consideradas. Os erros sistemáticos relacionados aos entrevistados foram minimizados através da exclusão de indivíduos cujos responsáveis não estavam aptos a responder o inquérito. Apesar do treinamento dos entrevistadores, ainda podem ter ocorrido diferenças decorrentes da interação com o entrevistado. Entretanto, acreditamos que os resultados encontrados refletem a tendência que ocorre na população-alvo do estudo.

Apesar de o consumo médio de ferro encontrado ter sido equivalente à IDR, pouco mais da metade das crianças apresentou consumo superior 
TABELA 5. Consumo médio de nutrientes por tipo de refeição em 210 crianças de 12 a 18 meses, Rio de Janeiro, Brasil, 1993 ${ }^{a}$

\begin{tabular}{|c|c|c|c|c|c|c|c|c|c|c|c|c|c|c|c|c|c|c|}
\hline \multirow[b]{2}{*}{ Refeição } & \multicolumn{3}{|c|}{ Energia } & \multicolumn{3}{|c|}{ Proteína } & \multicolumn{3}{|c|}{ Ferro (Fe) } & \multicolumn{3}{|c|}{$\begin{array}{c}\text { Ferro } \\
\text { biodisponível }\end{array}$} & \multicolumn{3}{|c|}{ Cálcio } & \multicolumn{3}{|c|}{ Vitamina C } \\
\hline & $\mathrm{M}$ & DP & $\%$ & M & DP & $\%$ & M & DP & $\%$ & $M$ & DP & $\%$ & M & $\mathrm{DP}$ & $\%$ & M & DP & $\%$ \\
\hline Desjejum (n = 210) & 262 & 96 & 18 & 8,7 & 3,7 & 16 & 1,1 & 0,9 & 11 & 0,05 & 0,08 & 7 & 291 & 146 & 33 & 9 & 17 & 11 \\
\hline Colação (n = 150) & 128 & 100 & 8 & 3,3 & 3,6 & 6 & 0,7 & 0,7 & 7 & 0,03 & 0,05 & 5 & 70 & 97 & 8 & 15 & 30 & 17 \\
\hline Almoço $(n=210)$ & 299 & 141 & 20 & 15,8 & 9,5 & 27 & 3,5 & 33 & 2,1 & 0,30 & 0,25 & 39 & 63 & 58 & 8 & 30 & 39 & 33 \\
\hline Lanche $1(n=208)$ & 251 & 129 & 17 & 7,4 & 4,4 & 13 & 1,4 & 1,0 & 14 & 0,06 & 0,06 & 10 & 216 & 152 & 22 & 15 & 24 & 18 \\
\hline Lanche $2(n=64)$ & 200 & 138 & 12 & 6,2 & 5,7 & 10 & 1,1 & 1,0 & 11 & 0,06 & 0,09 & 7 & 136 & 163 & 12 & 13 & 24 & 12 \\
\hline Jantar $(n=210)$ & 254 & 133 & 17 & 13,2 & 8,7 & 22 & 3,0 & 2,2 & 27 & 0,27 & 0,27 & 32 & 64 & 86 & 7 & 23 & 30 & 24 \\
\hline Ceia 1 (n= 169) & 239 & 106 & 16 & 7,6 & 3,8 & 13 & 1,0 & 0,8 & 10 & 0,04 & 0,03 & 6 & 258 & 166 & 24 & 7 & 12 & 10 \\
\hline Ceia 2 (n = 53) & 264 & 115 & 15 & 9,3 & 11,9 & 13 & 1,2 & 1,6 & 12 & 0,07 & 0,17 & 10 & 234 & 157 & 19 & 7 & 9 & 146 \\
\hline
\end{tabular}

a $\mathrm{M}=$ média; $\mathrm{DP}=$ desvio padrão.

a $10 \mathrm{mg}$ por dia. Assim, a alta média encontrada é justificada pelo consumo médio elevado de ferro por parte de poucas crianças. O consumo médio de ferro encontrado - 10,7 mg por dia foi superior ao relatado por Sichieri et al. (6,2 mg por dia) e por Silva $(7,3 \mathrm{mg}$ por dia) $(6,7)$.

No presente estudo, o consumo de ferro, em termos de sua adequação à IDR, foi semelhante ao encontrado em outros estudos. Silva (27) encontrou, em uma região rural da Bahia, consumo de ferro abaixo do nível recomendado pela IDR em $55 \%$ da amostra. Szarfarc et al. (25) observaram, no Município de São Paulo, que 48\% das dietas eram inadequadas em ferro; em apenas $14 \%$ dos casos totais a IDR de $10 \mathrm{mg}$ por dia foi atingida $(17 \%$ para a faixa etária de 12 a 24 meses). Sigulem (3), estudando crianças de 6 a 60 meses no Município de São Paulo, encontrou inadequação de consumo de ferro em 93\% das crianças na faixa etária de 6 a 24 meses. Uma vez que não foi encontrada, no presente estudo, inadequação de consumo energético, pode-se concluir que a inadequação de consumo de ferro não está relacionada a um baixo consumo alimentar, mas a uma baixa qualidade da composição alimentar. Este achado enfatiza a importância de ações educativas envolvendo famílias e comunidades. Observando-se os alimentos presentes no recordatório de 24 horas, é possível verificar que eram escassas as fontes dietéticas de ferro, e que estavam presen- tes combinações inadequadas que poderiam reduzir a absorção de ferro.

Ao avaliar-se a biodisponibilidade de ferro, um número ainda menor de crianças atingiu o requerimento de 1 mg por dia, demonstrando que a absorção de ferro estava comprometida para a maioria das crianças em função do baixo consumo de ferro heme (de origem animal) ou baixa absorção do ferro não-heme consumido. Estes resultados mostram que as dietas não supriam as necessidades de ferro da maioria da amostra estudada. O consumo protéico médio, apesar de adequado, não contribuiu para melhorar a biodisponibilidade do ferro e, provavelmente, deveu-se ao grande consumo de leite.
A associação entre baixo consumo de ferro biodisponível e anemia reflete uma composição de refeição imprópria para a manutenção de um adequado estado nutricional de ferro, sendo o consumo de ferro total menos importante do que a combinação dos alimentos na determinação da anemia. Pisacane (33), em 1995, também não encontrou diferenças significativas entre crianças italianas anêmicas e não anêmicas em relação ao consumo médio de ferro total. Portanto, a composição da dieta deve ser baseada na presença de alimentos ricos ou medianamente ricos em ferro, evitando ao máximo a presença de alimentos que inibem a absorção do ferro e buscando incluir potencializadores de sua ab-

TABELA 6. Associação entre combinações de alimentos presentes no almoço e anemia em crianças de 12 a 18 meses, Rio de Janeiro, Brasil, 1993

\begin{tabular}{|c|c|c|c|c|c|c|}
\hline \multirow[b]{3}{*}{ Alimentos } & \multicolumn{4}{|c|}{ Anemia $^{a}$} & \multirow[b]{3}{*}{$\mathrm{RP}(\mathrm{IC} 95 \%)^{\mathrm{b}}$} & \multirow[b]{3}{*}{$P$} \\
\hline & \multicolumn{2}{|c|}{ Presente } & \multicolumn{2}{|c|}{ Ausente } & & \\
\hline & No. & $\%$ & No. & $\%$ & & \\
\hline Sem carne & 29 & 46 & 34 & 54 & $0,88(0,65-1,20)$ & 0,39 \\
\hline Com carne & 77 & 52 & 70 & 48 & & \\
\hline Ferro não-heme ${ }^{c}$ sem fruta & 22 & 56 & 17 & 44 & $1,48(0,80-2,73)$ & 0,17 \\
\hline Ferro não-heme com fruta & 8 & 38 & 13 & 62 & & \\
\hline Ferro não-heme com leite de vaca & 7 & 70 & 3 & 30 & $1,49(0,90-2,45)$ & 0,18 \\
\hline Ferro não-heme sem leite de vaca & 24 & 47 & 27 & 53 & & \\
\hline Com LV & 13 & 62 & 8 & 38 & $1,23(0,85-1,76)$ & 0,32 \\
\hline Sem LV & 95 & 51 & 94 & 50 & & \\
\hline
\end{tabular}

a Anemia: $\mathrm{Hb}<11 \mathrm{~g} / \mathrm{dL}$.

b $\mathrm{RP}=$ razão de prevalências; $\mathrm{IC}$ = intervalo de confiança.

c Ferro de origem vegetal. 
TABELA 7. Freqüência de consumo de alimentos ricos em ferro em 278 crianças de 12 a 18 meses, Rio de Janeiro, Brasil, 1993

\begin{tabular}{|c|c|c|c|c|c|c|c|c|c|c|c|}
\hline \multirow[b]{3}{*}{ Alimentos $^{a}$} & \multicolumn{11}{|c|}{ Freqüência } \\
\hline & \multicolumn{2}{|c|}{1 a 2 vezes/dia } & \multicolumn{2}{|c|}{4 a 6 vezes/semana } & \multicolumn{2}{|c|}{1 a 3 vezes/semana } & \multicolumn{2}{|c|}{$<1 \mathrm{vez} /$ semana } & \multicolumn{2}{|c|}{ Nunca ofereceu } & \multirow{2}{*}{$\frac{\text { Total }}{\text { No. }}$} \\
\hline & No. & $\%$ & No. & $\%$ & No. & $\%$ & No. & $\%$ & No. & $\%$ & \\
\hline Feijão (caldo) & 26 & 10 & 0 & 0 & 8 & 3 & 0 & 0 & 238 & 88 & 272 \\
\hline Hortaliças folhosas & 28 & 10 & 3 & 1 & 110 & 40 & 18 & 7 & 119 & 43 & 278 \\
\hline Gema de ovo & 0 & 0 & 0 & 0 & 30 & 11 & 2 & 1 & 236 & 88 & 268 \\
\hline Carne bovina & 10 & 4 & 9 & 3 & 231 & 83 & 7 & 3 & 21 & 8 & 278 \\
\hline Fígado & 1 & 0 & 1 & 0 & 131 & 47 & 73 & 26 & 72 & 26 & 278 \\
\hline Sangue de ave & 0 & 0 & 0 & 0 & 16 & 6 & 8 & 3 & 254 & 91 & 278 \\
\hline
\end{tabular}

a Gema de ovo e caldo de feijão apresentaram $n<278$ devido à ausência de respostas para estes alimentos em alguns inquéritos.

sorção, especialmente para crianças de 6 a 24 meses, como forma de prevenção da anemia ferropriva.

Os alimentos mais consumidos no desjejum, colação e lanche justificaram os achados de baixo teor de ferro e baixa biodisponibilidade de ferro nestas refeições, de forma semelhante aos resultados de Tudisco et al. (34) e Silva (27). As grandes refeições foram de maior relevância para o fornecimento do ferro, pois, além de conterem ferro heme, apresentaram maior teor de vitamina C e menor teor de cálcio. Entretanto, foi encontrada baixa utilização de feijão e maior utilização de leite no jantar, o que contribui para a redução do ferro biodisponível. Alimentos que contêm nutrientes que reduzem experimentalmente a absorção do ferro praticamente não estavam presentes nas grandes refeições da amostra estudada, como o leite, queijo, requeijão, chocolates, café, chá e refrigerantes.

TABELA 8. Associação entre freqüência de consumo de alimentos ricos em ferro heme (origem animal) e ferro não-heme e anemia em crianças de 12 a 18 meses, Rio de Janeiro, Brasil, 1993

\begin{tabular}{|c|c|c|c|c|c|c|}
\hline \multirow{3}{*}{$\begin{array}{l}\text { Adequação da } \\
\text { freqüência de consumo }\end{array}$} & \multicolumn{4}{|c|}{ Anemia $^{a}$} & \multirow{3}{*}{$\begin{array}{c}\mathrm{RP}^{\mathrm{c}} \\
(\mathrm{IC} 95 \%)^{\mathrm{c}}\end{array}$} & \multirow[b]{3}{*}{$P$} \\
\hline & \multicolumn{2}{|c|}{ Presente } & \multicolumn{2}{|c|}{ Ausente } & & \\
\hline & No. & $\%$ & No. & $\%$ & & \\
\hline \multicolumn{7}{|l|}{ Feijão } \\
\hline Inadequada & 45 & 50 & 45 & 50 & 1,01 & 0,93 \\
\hline Adequada & 93 & 50 & 95 & 51 & $(0,79-1,30)$ & \\
\hline \multicolumn{7}{|l|}{ Hortaliças folhosas } \\
\hline Inadequada & 127 & 51 & 122 & 49 & 1,34 & 0,18 \\
\hline Adequada & 11 & 38 & 18 & 62 & $(0,83-2,18)$ & \\
\hline \multicolumn{7}{|l|}{ Carne bovina/aves } \\
\hline Inadequada & 116 & 51 & 112 & 49 & 1,16 & 0,37 \\
\hline Adequada & 22 & 44 & 28 & 56 & $(0,82-1,62)$ & \\
\hline \multicolumn{7}{|l|}{ Fígado } \\
\hline Inadequada & 67 & 47 & 77 & 54 & 0,87 & 0,23 \\
\hline Adequada & 72 & 54 & 62 & 46 & $(0,68-1,10)$ & \\
\hline
\end{tabular}

a Anemia: $\mathrm{Hb}<11 \mathrm{~g} / \mathrm{dL}$.

b Em relação à ingestão dietética recomendada.

c $\mathrm{RP}=$ razão de prevalências; $\mathrm{IC}=$ intervalo de confiança.

A ocorrência de anemia em crianças que consumiram refeições sem frutas confirmou os achados experimentais relativos à absorção de ferro na presença de vitamina $C$ e está de acordo com os achados de Uchimura (26), que também encontrou associação entre a anemia e a ausência de frutas em crianças do Estado do Paraná. Não se encontrou, no presente estudo, associação estatisticamente significativa entre a ocorrência de anemia e vários fatores alimentares que supostamente causariam anemia, como por exemplo a presença de cálcio na refeição.

Vale ressaltar que a validade do recordatório de 24 horas no referido trabalho é a determinação de uma clientela com riscos para apresentar ou agravar a anemia, pois os resultados procedentes deste tipo de inquérito refletem o consumo atual, e não o consumo alimentar que pode ter gerado o quadro encontrado de deficiência de ferro.

\section{CONCLUSÕES}

Uma vez que a composição da dieta, e não a quantidade da alimentação, é o elemento que parece condicionar a deficiência de ferro, ações de caráter educativo, neste caso, não podem ser desconsideradas. Em particular, é importante pensar em ações que enfatizem a combinação dos alimentos nas 
grandes refeições, aumentando o teor de ferro através da utilização de alimentos como cereais e leite fluido. Nas grandes refeições, é pouco razoável esperar aumento no consumo de ferro heme, encontrado em alimentos caros, como a carne. Conseqüentemente, os esforços devem ser concentrados no aumento do consumo e biodisponibilidade do ferro não-heme.

Neste contexto, o componente educativo consiste basicamente no conhecimento sobre inibidores e facilitadores da absorção do ferro e depende da ação dos profissionais de saúde. A estratégia de Atenção Integrada às Doenças Pre- valentes na Infância (35) pode contribuir de maneira importante neste sentido, pois contempla, em suas normas, orientações alimentares gerais apropriadas, além de adaptadas às realidades locais, podendo incluir orientações alimentares específicas para o tratamento e a prevenção da anemia ferropriva.

\section{REFERÊNCIAS}

1. World Health Organization. Infant feeding the physiological basis. Bull World Health Organ 1989:67(Suppl).

2. International Nutritional Anemia Consultative Group (INACG). Guidelines for the eradication of iron deficiency anemia: a report of the International Nutritional Anemia Consultative Group. Washington, DC: INACG; 1979.

3. Sigulem DM, Tudisco ES, Goldemberg P, Athaide MM, Vaisman E. Anemia ferropriva em crianças do município de São Paulo. Rev Saude Publica 1978;12(2):168-78.

4. Monteiro CA, Sczarfarc SC. Estudo das condições de saúde das crianças no Município de São Paulo, SP (Brasil), 1984-1985. V - Anemia. Rev Saude Publica 1987;21(3):255-60.

5. Araújo RL, Araújo MBDG, Sieiro RO, Machado RD, Leite BV. Diagnóstico de hipovitaminose A e anemia nutricional. Estudo realizado na população do Vale do Jequitinhonha, Minas Gerais. Rev Bras Med 1986 43(8):225-8.

6. Sichieri R, Szarfarc SC, Monteiro CA. Relação entre dieta e ocorrência de anemia ferropriva em crianças. J Pediatr (Rio de J) 1988;64(5): 169-74.

7. Lozoff B, Jimenez E, Wolf AW. Long-term developmental outcome of infants with iron deficiency. N Engl J Med 1991;325(10):687-94.

8. Lozoff B, Klein NK, Nelson EC. Behavior of infants with iron-deficiency anemia. Child Dev 1998;69(1):24-36.

9. Hallberg L, Brune M, Rossander L. Effect of ascorbic acid on iron absorption from different types of meals. Human Nutr Appl Nutr 1986;40(2): 97-113

10. Gillooly M, Torrance JD, Bothwell TH, MacPhail AP, Derman D, Mills W, et al. The relative effect of ascorbic acid on iron absorption from soy-based and milk-based infant formulas. Am J Clin Nutr 1984:40(3):522-7.

11. Charlton RW, Bothwell TH. Iron absorption. Annu Rev Med 1983;34:55-68.

12. Martinez-Torres C, Romano E, Layrisse M. Effects of cysteine on iron absorption in man. Am J Clin Nutr 1981;34(3):322-7.

13. Garcia-Casal MN, Layrisse M, Solano L, Beron MA, Arguello F, Llovera D, et al. Vita$\min \mathrm{A}$ and beta-carotene can improve nonheme iron absorption from rice, wheat and corn by humans. J Nutr 1998;128(3):646-50.

14. Rossander L, Hallberg L, Björn-Rasmussen E. Absorption of iron from breakfast meals. Am J Clin Nutr 1979;32:2484-9.
15. Hallberg L, Rossander L. Effect of different drinks on the absorption of non-heme iron from composite meals. Human Nutr Appl Nutr 1982;36(2):116-23.

16. Siegenberg D, Baynes RD, Bothwell TH, Macfarlane BJ, Lamparelli RD, Car NG, et al. Ascorbic acid prevents the dose-dependent inhibitory effects of polyphenols and phytates on nonheme-iron absorption. Am J Clin Nutr 1991;53(2):537-41.

17. Woods S, DeMarco T, Friedland M. Iron metabolism. Am J Gastroenterol 1990;85(1):1-8.

18. Brune M, Rossander-Hultén L, Hallberg L, Gleerup A, Sandberg AS. Iron absorption from bread in humans: inhibiting effects of cereal fiber, phytate and inositol phosphates with different numbers of phosphate groups. J Nutr 1992;122(3):442-9.

19. Lynch SR, Dassenko SA, Cook JD. Inhibitory effect of a soybean-protein-related moiety on iron absorption in humans. Am J Clin Nutr 1994;60(4):567-72.

20. Hallberg L, Rossander-Hultén L, Brune M, Gleerup A. Calcium and iron absorption: mechanism of action and nutritional importance. Eur J Clin Nutr 1992;46(5):317-27.

21. Hallberg L, Brune M, Erlandsson M, Sandberg AS, Rossander-Hulten L. Calcium: effect of different amounts on nonheme- and hemeiron absorption in humans. Am J Clin Nutr 1991;53(1):112-9.

22. Hallberg L, Rossander-Hultén L, Brune M, Gleerup A. Bioavailability in man of iron in human milk and cow's milk in relation to their calcium contents. Pediatr Res 1992;31(5):524-7.

23. Gleerup A, Rossander-Hultén L, Hallberg L. Duration of the inhibitory effect of calcium on non-haem iron absorption in man. Eur J Clin Nutr 1993;47(12):875-9.

24. Calvo EB, Gnazzo N. Prevalence of iron deficiency in children aged 9-24 mo from a large urban area of Argentina. Am J Clin Nutr 1990;52(3):534-40.

25. Szarfarc SC, Monteiro CA, Meyer M. Estudo das condições de saúde das crianças do Município de São Paulo, SP (Brasil), 1984/1985. X. Consumo alimentar. Rev Saude Publica 1988; 22(4):266-72.

26. Uchimura TT. Anemia e desnutrição em escolares ingressantes nas escolas estaduais de Maringá, PR [dissertação]. São Paulo: Faculdade de Saúde Pública, Departamento de Nutrição da Universidade de São Paulo; 1994.
27. Silva RDCR. Determinantes da anemia em população infantil da zona rural do semi-árido baiano [dissertação]. São Paulo: Faculdade de Ciências Farmacêuticas e Faculdade de Economia da Universidade de São Paulo; 1993.

28. SISNUT — Sistema de Nutrição [programa de computador]. Rio de Janeiro: Pyrrho AS \& Lacerda EMA; 1993.

29. Food and Agriculture Organization/Organizacion Mundial De La Salud. Necesidades de energia y proteinas: Informe de una reunión consultiva. Serie de Informes Técnicos. Genebra: FAO/OMS; 1985.

30. Food and Agriculture Organization/Organizacion Mundial De La Salud. Necesidades de vitamina A, hierro, folato y vitamina B12: Informe de una consulta mixta de expertos. Roma: FAO/OMS; 1991

31. National Research Council/Food and Nutrition Board/National Academy of Sciences. Recommended Dietary Allowances. 10th ed. Washington DC: NRC/FNB; 1989.

32. EPI-INFO 6.04a: A word processing database and statistics program for public health on IBM-compatible microcomputers [programa de computador]. Atlanta: Centers for Disease Control and Prevention/World Health Organization; 1996.

33. Pisacane A, De Vizia B, Valiante A, Vaccaro F, Russo M, Grillo G, et al. Iron status in breastfed infants. J Pediatr 1995;127(3):429-31.

34. Tudisco E, Marin P, Shrimpton R, Costa M, Donohue R. Alimentação no desmame em áreas periurbanas de quatro capitais brasileiras: resultados preliminares. J Pediatr (Rio de J) 1988;64(6):231-6.

35. Pan American Health Organization. Atenção Integrada às Doenças Prevalentes na Infância [site da Internet]. www.paho.org/portuguese/gov/ce/ce124_14.pdf. Acessado em 7 de maio de 2001

Manuscrito recebido em 19 de outubro de 2000. Aceito em versão revisada em 12 de abril de 2001 
ABSTRACT Objective. To assess the influence of feeding practices on the prevalence of anemia among breast-fed children, based on the nutritional profile of children receiving care at an outpatient pediatric clinic in the city of Rio de Janeiro, Brazil.

Iron deficiency anemia and Methods. This was a cross-sectional study with 288 children between 12 and 18 months of age who were seen at the outpatient clinic between January and June 1993. The children were assessed in terms of the presence of iron-deficiency anemia. In addition, two questionnaires were done with the child's mother or the other person who had brought the child to the clinic: a 24-hour dietary recall and a record of the frequency of the consumption of iron-rich foods.

Results. Of the 288 children, 144 of them had signs of anemia (hemoglobin $<11 \mathrm{~g} /$ $\mathrm{dL}$ ); of these 144, 38 of them had severe anemia (hemoglobin $<9.5 \mathrm{~g} / \mathrm{dL}$ ). We found low levels of bioavailable iron in the foods consumed, limited consumption of meat, and vitamin $C$ consumption separate from meals. We found a significant association between the prevalence of severe anemia and inadequate iron intake (relative risk = 2.28; $95 \%$ confidence interval $=1.12$ to $4.66 ; P=0.02$ ). The intake of bioavailable iron was higher in the group without anemia $(P=0.04)$.

Conclusions. Individuals caring for breast-fed children should be educated concerning the nutritional contents of complementary foods so as to increase the bioavailability of iron in the children's diets. One way to achieve this objective might be through "Integrated Management of Childhood Illnesses," a strategy endorsed by a number of international organizations as a way to reduce child mortality and morbidity in developing countries.

\section{Cursos monográficos sobre alimentación y nutrición (2001-2002)}

Fechas: $\quad$ octubre de 2001 a mayo de 2002

Institución responsable: Universidad de Navarra, Pamplona, España

El interés por la alimentación y la nutrición está cobrando auge debido a su estrecha relación con el estado de salud del ser humano y con la prevención y el tratamiento de enfermedades que afectan a millones de personas en el mundo entero. Resulta cada vez más evidente la necesidad de dar mayor cabida a estos temas en el plan de estudios de los médicos y otros profesionales de la salud.

La enseñanza a distancia y en línea ofrece a quienes se dedican al ejercicio de su profesión la oportunidad de actualizarse sin descuidar su actividad laboral cotidiana. Con tal meta, la Universidad de Navarra ofrece seis cursos de educación continuada a distancia sobre alimentación y nutrición: 1) Ciencia de los Alimentos, 2) Nutrición y Salud Pública, 3) Alimentos: Composición y Propiedades, 4) Nutrición en la Infancia y la Adolescencia, 5) Nutrición y Obesidad y 6) Nutrition and Obesity. Estos cursos, que se impartirán consecutivamente de octubre de 2001 a mayo de 2002 (por orden de mención), combinan la formación teórica tradicional con los métodos didácticos y medios informáticos e interactivos más modernos (consultas por fax, teléfono o correo electrónico; videoconferencias; autoevaluación por la Internet, etc. Todos tienen una duración de 50 horas, salvo el último, que dura 25 horas. Los cursos están diseñados para llevarse a cabo desde el lugar donde reside el alumno, con o sin el empleo de una computadora. Puede inscribirse cualquier persona con título universitario (licenciada o diplomada) que radique en España o en otro país. Debido al número limitado de plazas, se recomienda matricularse temprano, teniendo presentes las siguientes fechas límite para la matrícula de cada curso (en orden de mención): 3 de septiembre, 11 de octubre y 30 de noviembre de 2001, y 28 de enero, 18 de febrero y 23 de febrero de 2002.

\section{Información:}

Tel.: (34)-948-425665 (directo) ó (34)-948-425600 (extensión 6432)

Horario de atención telefónica (en España): 11.30 a 13.30 h, lunes a viernes

Fax: (34)-948-425649

Correo electrónico: nutdist@unav.es

Internet: http://www.unav.es/farmacia/graduados/cdistns/ 